\title{
Ptychography: how it has developed, and where it might lead
}

\section{Chris Jacobsen}

\author{
Argonne Lab/Northwestern University, Argonne, United States of America; \\ cjacobsen@anl.gov
}

The idea for ptychography dates back to 1969 , but its realization as a practical imaging method awaited the development of iterative phase retrieval algorithms. By now, it is firmly established for nanoscale studies of materials using X rays, both in transmission mode and also using Bragg diffraction. While focusing optics greatly aid its implementation, the spatial resolution is determined not by optics but by the finest length scales from which one can measure elastic scattering. On the experimental side, the hundredfold increases in quasi-time-continuous coherent flux provided by diffraction-limited storage rings will dramatically advance what ptychography can do. On the computational side, the application of nonlinear optimization approaches has allowed one to compensate for many experimental limitations, including errors in nanopositioning as well as partial coherence, and allow one to re-think how one might acquire ptychographic data. Thus far, $\mathrm{x}$-ray ptychography has been applied to millimeter-size samples in $2 \mathrm{D}$, and roughly 10 micrometer size samples in 3D. How far might that go? Can one combine the advantages of $\mathrm{X}$ rays of high penetration power and low multiple scattering to image even larger samples? Can one carry out nanoscale imaging of cubic centimeter volumes? I outline some of the opportunities this might provide, and some of the challenges in achieving this.

Keywords: X-ray ptychography, x-ray microscopy, nanoscale imaging 